\title{
The Information/Knowledge society as Risk Society : Assessing and Enforcing IT safety and security standards for IT systems : about responsibility of experts and governments
}

\author{
Klaus Brunnstein \\ Faculty for Informatics, University of Hamburg, Germany \\ brunnstein@informatik.uni-hamburg.de \\ http://agn-www.informatik.uni-hamburg.de
}

\begin{abstract}
As daily experience demonstrates, contemporary networks (esp The Internet) and computer systems (whether clients or servers) are "Insecure and Unsafe at any speed" (quotation from Ralph Nader, addressing industrial products). Besides many beneficial effects of IT for enterprises, organisations and individual, many incidents have contributed to significant loss and damage. Even pubertarian boys succeed easily in attacking important IT systems and produce significant damage to systems, users and customers. Among several reasons, including basic design of technologies, IT experts do not care sufficiently for the consequences of their design, products and usage. As technical improvements of contemporary IT systems will - for a foreseeable period - only partly help to overcome basic causes of InSecurity, education of IT engineers to safer and more secure design and implementation of their products may help to reduce IT risks. While some professional organisations such have suggested some rules for ethical behaviour of their members, contemporary curricula fail to include Ethics into the education of IT experts esp. Including System and Software Engineering. "Good Practice" becomes even more important with growing dependency of enterprises, organisations, governments and individuals on vulnerable and growingly interconnected IT systems, IT and legal experts must find ways to enforce "good practice".
\end{abstract}

Keywords: systems security, vulnerability, computer professionals, user empowerment, democracy 


\section{Evolution of Digital Technologies (DTs) in "Information and Knowledge Society (IS/KS)"}

In the history of mankind, several technologies have contributed to change economic and individual conditions and perspectives. While most technical inventions - such as horse-driven cars, windmills and ships - developed rather slowly concerning technology as well as regarding their impact on society, James Watts invention (1761) of the vapour pressure driven "machine" changed economic conditions and in their wake the world order at significantly higher pace than any technology before. In their first - stationary - form, production of industrial goods was operated by heavy machines with relatively small power (though large compared to human "manufacturing") but it was the start to essentially change the production of related goods. This change gained more speed and had more effects when Watts machines became sufficiently light and more powerful as to drive wagons on iron rails ("locomotion") to support faster transport of material resources to the fabrication sites and of products to consumers. Both the stationary and the mobile machine supported developments of the "Industrial Society" which gained more momentum with other technical inventions and developments such as electrical energy, telephony and the energy production from resources such as oil and uranium.

When comparing economies and state organisation, law, education and the role of individuals between the advent of Watts engine and 1960 when computers began to significantly spread into (then large) business and universities, then economic and societal changes are evident, at least in the so called (technically) "developed countries". And changes affected many areas: from hierarchical to democratic organisations, legal systems supporting individual rights, opportunities for education and medical support for all, etc. But over the span of about 200 years of industrial history (1762-1960), the speed of change was rather slow.

As basis of predicting future developments of ICTs, an analysis of similarities and differences in developments of industrial technologies may help. Concerning economic effects on political economies, economical theories (Schumpeter, Kondratieff et al) have observed cyclic behaviours in Industrial economies where new technologies stimulated economic growth. When concentrating on essential ("lead") technologies, industrial developments can be classified into essentially 4 cycles, each with about $40-50$ years of duration ( $\sim 45$ years).

Faster than industrial technologies before, Information and Communication technologies (ICTs) continue to change economies and societies in ways affecting many aspects of human lives. "Traditional" communication - telephony using terrestrial cables, satellite and mobile cell-based communication - has enlarged human communication space even in its previously analogous, now also digitalized forms. Cables are still the major basis of digital networks but in some areas, flexible mobile communication is a major factor of information exchange in enterprises and daily life.

Concerning "lead technologies", it is interesting that phase 1 of the Information Economies depended upon initially large, heavy and difficult to handle "engines" (mainframes and their software) operated by specialists in "computer centres" similar to factories in the Industrial economies - which developed into smaller and 
The Information/Knowledge society as Risk Society :

for IT systems : about responsibility of experts and

governments

more powerful machines (Mini, Micro and Personal Computers). With their advent, transport of digital results between remote sites became important and networks developed both within enterprises (Intranets, Local Area Networks) and globally. The development of The Internet - designed and intended for scientific and military communication - became the major "lead" technology for the 2 nd phase, similar to the development of railway transport in the 2nd phase of the Industrial Age.

In the Industrial age, railways enabled faster transport of resources and products as well as they also supported human transport on broad scales. Thus, railways changed economic and human relations as they enabled to progress from local to regional and finally global activities. In similar ways, Information and Communication technologies have started to rapidly and deeply affect many relations established within the Industrial societies.

From Local-Area Networks (LANs) to the Internet as a cooperation of many Wide-Area Networks (WANs), digital communication based on the uniform Internet communication Protocol (IP) is changing many relations in and between enterprises (Business to Employee, B2E; Business to Business, B2B), but also between business and customers (business to customer, B2C) and governments (B2G). In same sense, though with lower speed of development, individual relations as patient (Health to Patient, H2P), student (School to Student, S2S, Student to University, S2U) and citizen (Citizen to Government, C2G) use technical features of digital communication combined with storage and processing of information. Essential processes of the Information and Communication Societies will be based upon production of digital values such as Information Search (aka Data Mining, I-Search): Changes in education such as distant and e-Learning as well as in democratic processes, e.g. e-voting are underway.

Remark: following contemporary implementation, such processes are labelled "e-"for "electronic", thus indicating the implementation using electromagnetic media and processes. As future technologies will likely use photonic and quantum technologies, it seems advisable to label such relations as "d-relations" thus addressing their digital nature.

Admittedly, speed and impact of these developments vary grossly over the planet. As in the Industrial Age, several countries (better: regions within nations) advance technical developments rapidly and benefit from their advantages esp. including the transfer of related products and methods into other parts of the world. The uneven distribution of development between regions and nations also implies that e-relations develop rather differently over the planet. With respect to the opportunities of modern technologies, the "digital gap" hinders less "developed areas" to participate in this global process for the best of their people. On the other side, this "digital gap" protects less-developed areas to be affected by unwished sideeffects and serious (e.g. security) problems of these technologies which affect enterprises, governments, organisations and individuals growingly with undesired effects. 


\section{Risk Analysis of emerging Digital Technologies}

After short times of usage of these technologies, organisations and individuals have become so dependant upon proper functioning of these highly complex and hardly understandable systems that any deviation from "normal" behaviour may have adversary if not damaging effects. It belongs to the daily experiences of "users" of Information and Communication Technologies that computerized systems fail rather often for reasons which a "normal user" can hardly analyse or understand. Failures range from unforeseen crashes, infections with malicious software esp. imported from Intranet or Internet communication to loss of data and programs and to a complete loss of any digital function and of connectivity.

A Risk Analysis of contemporary ICTs reveals a variety of reasons why such systems fail to work properly or to work at all. Following the "life cycle" of ICT concepts and products, risks can be graded into "vulnerability classes" which range from paradigms dominating their design - paradigmatic risks - over risks from deficiencies in the quality of production and products - implementation risks - to risks in ways how systems are used - usage risks. In addition, ICTs may be deliberately misused for criminal purpose.

Paradigmatic risks (Vulnerability Class 1): risks in this class are deeply inherent in assumptions made in the design process, and in the methods applied in the production (aka implementation) of hardware, systems and application software. Assumptions range from the concept that complex problems can be solved by cutting them into parts ("modules") which can be produced independently and subsequently combined to yield systems of high complexity. Apart from problems of adequate "cutting" of the modules and of their adequate interoperation, contemporary systems have become so complex that even experts can hardly understand their effects. In order to archive more functionality and interoperability, complex systems are combined with others to produce even higher grades of complexity. When systems from different origins with no common "interface" are combined, instruments are needed to "glue" such systems together; such "glue-ware" - "script" programming languages such as Java or Virtual BASIC - must be powerful to achieve many different adaptations which also provide easy means of exploitation even by less qualified "script kiddies". Indeed, the plenitude of malicious software (computer viruses and network worms, Trojan horses, trapdoors, backdoors, spy-ware etc) is essentially based on script languages used e.g. in office systems (Visual Basic for Applications etc). In summary, concepts and tools used in the design process very deeply influence both functions and risks of these digital technologies.

Risks from inadequate implementation (Vulnerability Class 2): the production of digital technologies, esp. system and application software (and to a lesser degree also of hardware and their "drivers") has many weaknesses, the most evident of which are inadequate qualification of programmers, inadequate testing and production under heavy time pressure. Undesirable results of conceptual and programming faults materialize mostly at user sites where such software behaves in unpredictable manners including destruction of achieved work and broken 
The Information/Knowledge society as Risk Society :

Assessing and Enforcing IT safety and security standards

for IT systems : about responsibility of experts and

governments

connections. Numbers of experienced "computer emergencies" are rapidly growing, with sometimes millions of servers and even more local computers being affected by software weaknesses and "infected" by network "worms".

Software weaknesses are inherent in all kinds of contemporary software but the predominant effects materialize on systems of the most dominant system and software producers. Here, Microsoft leads both the markets of sold software and the world of software flaws as well as of malicious software living upon Microsofts insecure software design and imperfect software implementation. Although there are also many problems with non-MS systems (e.g. Linuxes), Microsoft systems dominate the scene of those incidents where in some cases many millions computer systems were affected.

Risks from usage (Vulnerability Class 3): not surprisingly, usage of unsafely designed and insecurely implemented software presents additional risks. After distributed software reaches user sites, installation and administration of system and application software when improperly performed may adversely affect performance and proper functioning of such software. Due to the complexity as well as due to inadequate documentation of these systems, users hardly understand effects of their attempts to "properly" use such systems. Consequently, users apply "trial and error" methods in learning to work with new features, rather than trying methodologically to understand which functions may have which effects, and which precautions should be taken to avoid unwished side-effects. This somewhat "explorative" way to use systems rather often leads to a risky attitude with potentially hazardous effects, e.g. by clicking on unknown attachments without due care.

Software manufacturers often argue that failure of software is mainly caused by improper actions of users. But in many - if not most - cases, the human-computer interface (e.g. the display of functions and operations on the screen, or the handling of input devices such as mouse and keyboard) are inadequately designed and users are not properly supported by help functions (which when existing in many cases are so complex that users are further mislead). While users are primarily interested to do their work, one must admit that they rather often tend to forget about any precaution and even sometimes bypass security measures when thinking that their work performance is reduced.

Risks from deliberate misuse (Vulnerability Class 4): Digital Information and Communication technologies provide many opportunities for deliberate misuse including criminal purposes. Though only few cases of criminal misuse have been reported and prosecuted, some of which were broadly covered in media although few of which produced major damage (such as the "SoBig" worm affecting some 100 millions of emails and several 10.000 enterprise servers), deliberate misuse for criminal purposes has not yet reached a significant impact on business and government use. Consequently, both legal provisions and the ability of prosecution agencies is less developed than in other areas of criminal law. But there is no doubt, that further development of ICTs will be associated with growing misuse, esp. as long as Class 1 and Class 2 vulnerabilities are so dominant in contemporary ICTs. 


\section{Impact of Vulnerabilities on Information and Knowledge Societies}

As industrial technologies in the industrial age, Information and Communication Technologies will unavoidably affect many (though not all) parts of human organisations, economies, government and individual lives. As in the industrial society, these technologies are driven by supply-side concepts, and an analysis of any impact upon customers is (too) rarely in the scope of ICT developers. Consequently, such impact comes over the users - which have rarely any choice of avoiding ICT applications - without any possibility for them to understand or contain unwished effects.

Some of these changes have materialized in the first phase of the Information Society (based upon stationary operation), such as dependability from complex systems: as nobody can control whether results are correct in any detail, a consciousness of blind reliance among users has developed: "this must be true because this was produced by a computer". This pattern of overreliance and risk acceptance are even now, at the beginning of the 2nd phase (network-based operation) dominant. Comparable to those animals (lemmings) which blindly follow their forerunners, users tend to accept risks of PCs and The Internet as they feel unable to avoid them. In some sense, the more technologically a society advances, the more risks are blindly accepted. This seems to be a general pattern in contemporary societies as sociologists and philosophers observe and even label them as "risk societies" (Beck).

Besides general impact such as acceptance of risk, impact on individual attitude and behaviour can also be observed. While email as tool of direct and fast communication supports personal and business needs very well, at the same time it enforces an adaptation in user behaviour. At best, email enforces fast reaction and thus tends to dominate the time management of users: if you await fast reaction, you must also react fast, independent whatever else you are doing. Replying to email resets any other priority. This becomes esp. critical when unwished email seems to require reaction, such as malicious software requesting sanitary actions or floods of unwished email (spamming).

In general, usage of time changes significantly with contemporary communication systems. Similar to workers in industrial factories, users in connected ICT systems behave as slaves of engines which they can hardly understand and control. It remains a major task of education in the Information Age to unable users to master these engines rather than becoming controlled by them.

\subsection{Responsibility of Experts and Governments to Deploy Safe and Secure Digital Technologies}

In an Information Society so strongly depending upon complex and difficult to understand technologies, it is a primary responsibility of IT experts designing, implementing and operating related infrastructures and methods to avoid related risks as far as possible, and to make users aware of existing risks in order to help them to master related problems. Hence, risk assessment and risk management are major tasks for the forthcoming generations. 
The Information/Knowledge society as Risk Society :

for IT systems : about responsibility of experts and

governments

With "risk avoidance", Information and Knowledge systems must be structured in such a way that a class of given risks cannot materialize. Example: this strategy implies that a system is designed and constructed so that it cannot fail.

With "risk reduction", methods and mechanisms must be realized which reduce unwanted effects when some risk materializes, hopefully with lower probability. Example: this strategy implies that a system may fail but that there are curative mechanisms which reduce the damage when the system fails.

With "risk acceptance", nothing preventive or curative is done ("do not care" strategy). Example: although one knows about the vulnerability of a system, one simply hopes that nothing will happen and so one does nothing to prevent or reduce the risk.

While risk acceptance is what the vast majority of users practice, risk reduction is the strategy which many enterprises and governments presently apply: in order to reduce impacts of crashing systems and programs, "computer viruses" and "worms", hacker attacks, mass distribution of unwished email etc, special forms of security software (antivirus software, firewalls, intrusion detection systems etc) are deployed to reduce threats.

Risk acceptance may be regarded as acceptable strategy as long as large damage can be avoided. This strategy will be no longer acceptable when large damage cannot be avoided with minor protection methods. At the end of the 2 nd cycle of the Information Society, dependability will have reached a degree that many small failures combine to blackouts similar to (though more serious than) recent power outages in USA and Europe.

In order to avoid that networks become so strongly interconnected that any failure becomes "critical" for large parts of societies and economies, the only solution in the next decennium is to redesign basic technologies as to become inherently safe and secure. Regrettably, mankind will only learn - as in the Industrial Ages - by severe accidents how urgent the need for safe and secure system designs even today is.

Beyond technical solutions, additional measures such as regulation of required preventive actions as well as requirements for curative "post mortem" (that is: provisions when systems have severely failed) must be taken by government and regulating bodies. Besides regulations requiring security standards enforcing "best practice", additional regulations (directives, national and international laws) must enforce protection of customers (persons AND enterprises) in cases of failure or dysfunctional behaviour, esp. including compensation of costs and consequences of experienced damage. Here, a minimum amount of knowledge about Digital Technologies and their weaknesses must be available at the regulating bodies, and these bodies must protect users rather than supply side, implying some resistance against the interference of the (presently dominant) IT lobbies. Indeed, manufacturers of insecure systems and software must become liable for significant loss of data and time as consequence from crashes of their products. 


\section{References}

Alois Schumpeter Joseph (1939), Business Cycles: A Theoretical Historical and Statistical Analysis of the Capitalist Process, $1^{\text {st }}$ edition McGraw

Beck Ulrich (1992), Risk society: Towards a New Modernity, London, Sage

Berleur Jacques, Brunnstein, Klaus (ed) (1996), Ethics of Computing: Spaces for Discussion and Law, London, Chapman \& Hall.

Berleur Jacques, Brunnstein Klaus (ed) (2002), Human Choice and Computers: Issues of Choice and Quality of Life in the Information Society, Kluwer.

Brunnstein Klaus (1997), Towards a holistic view of security and safety of enterprise information and communication technologies: adapting to a changing paradigm, Kluwer 1997

Kondratieff Nikolai (1984), Long Wave Cycle, Richardson and Snyder.

Nader Ralph (1965), Unsafe at Any Speed: The Designed-In Dangers of the American Automobile, United Press International Baltimore 